\title{
Danzas folclóricas: una forma de aprender y educar desde la perspectiva sociocultural Folk dances: a way to learn and educate from the sociocultural perspective Michelle Matos-Duarte, Eulisis Smith, Alejandro Muñoz Moreno \\ Universidad Francisco de Vitoria (España)
}

Resumen: Estudiar y vivenciar la danza folclórica desde una perspectiva sociocultural (Conesa, 2017), es una oportunidad para acercar el alumnado al contexto de movimientos que cobran sentido como forma de expresión, insertados en las danzas de distintas partes del mundo. Además, es una importante herramienta para la instrucción de futuros maestros de Educación Física que suelen verse poco capacitados para la enseñanza de este contenido (Cañabate, Rodríguez \& Zagalaz, 2016). En este contexto, el objetivo planteado ha sido ofrecer una experiencia didáctica que aproxime al futuro profesorado de Educación Física al contenido de las danzas folclóricas, de tal manera que favorezca el conocimiento y la formación del mismo para la consecución del currículum. El proyecto se desarrolló en la asignatura de Actividades Físicas Artístico-Expresivas, con un total de 108 alumnos universitarios del grado de Ciencias de la Actividad Física y del Deporte. El diseño de la experiencia didáctica se dividió en: la construcción teórica, la vivencia práctica de danzas folclóricas de los cinco continentes y la experimentación de role play de los alumnos. A partir de esta experiencia dentro del aula universitaria se hizo una aproximación de los estudiantes con el contenido de danzas de distintos continentes. La vivencia teórico-práctico de danzas folclóricas es una alternativa a considerar, ya que colabora como herramienta educativa tanto para la formación y capacitación del profesorado de Educación Física como para el acercamiento del alumnado a la danza y su valor sociocultural.

Palabras clave: danza folclórica, danza educativa, interdisciplinar, sociocultural, educación física.

\begin{abstract}
Studying and experiencing folk dances from a sociocultural perspective (Conesa, 2017) is an opportunity for students to understand the context of movements that make sense as forms of expression, embedded into dances from different parts of the world. In addition, this is an important tool for the instruction of future Physical Education teachers, who do not usually see themselves as really in a position to teach this content (Cañabate, Rodríguez \& Zagalaz, 2016). In this context, the objective is to offer a didactic experience that brings future Physical Education teachers closer to the content of folk dances, in order to increase their knowledge about the subject and encourage training to complete the curriculum. The project was conducted in the subject of Physical Artistic-Expressive Activities, with a total of 108 undergraduates enrolled in the Physical Activity and Sports Sciences (CAFyD) degree. The design of the didactic experience was divided into: theoretical construction, practical experience of folk dances of the five continents, and experimenting role play with the students. From this experience, students within the university were introduced to the topic of dances from different continents. The theoretical-practical experience of folk dances is an alternative to be considered, as it contributes as an educational tool both for the training and qualification of Physical Education teachers and for the approach of students to dancing and its sociocultural value.
\end{abstract}

Keywords: folk dance, dance education, interdisciplinary, sociocultural, physical education.

\section{Introducción}

La danza entendida como una genuina expresión de los sentimientos humanos transmitidos en forma de movimientos, está arraigada en las costumbres y vivencias socioculturales de un determinado entorno geográfico. Por medio de la danza, el ser humano es capaz de reflejar y conciliar la complejidad de las múltiples dimensiones de la existencia (Porpino, 2018), conectando e interactuando de forma inherente con el otro y con uno mismo. Presente en distintas civilizaciones, es posible observar el uso pedagógico de la danza a lo largo de la historia (Fuentes, 2006), siendo capaz de transcender en el tiempo, inventándose y redescubriéndose a cada nueva generación. Igualmente, es un importante medio de comunicación no verbal a través del lenguaje corporal repleto de intencionalidad, sentidos y significados, capaz de desvelar la esencia y expresión que conforma una sociedad (Zandoná Júnior \& Silva, 2018).

La conexión entre lo corpóreo, lo sensible y lo estético desvelado en la danza permite la experiencia de acercamiento entre las personas. Entretanto, estas manifestaciones no suelen ser tratadas con la debida importancia que tienen. Según Porpino (2018), esto es debido al pensamiento educacional de occidente, el cual se caracteriza por una fuerte priorización de lo racional en detrimento de la sensibilidad,

Fecha recepción: 24-07-19. Fecha de aceptación: 08-01-20

Michelle Matos Duarte

michelle.matos@ufv.es que conlleva a la omisión o menosprecio de la belleza y el valor de las dimensiones que identifican y conforman la danza. Más allá de una simple expresión física del cuerpo a través de movimientos rítmicos y estéticos reproducidos, la danza permite la exteriorización de las emociones, sentimientos e ideas, favoreciendo la percepción, la comprensión y la reflexión del propio estado emocional (Rueda \& López, 2013), resultando ser un potente medio de comunicación con el mundo (Zandoná Júnior \& Silva, 2018) y una forma de transmitir un mensaje entre las distintas culturas. Hecho que la hace ser un instrumento relevante en el ámbito educativo para el trabajo del autoconocimiento, autoconcepto y un puente de conexión social con el otro que permite al alumnado vivir situaciones afectivas y sociales en distintos contextos (Domínguez \& Castillo, 2017; Gil-Madrona, Gutiérrez \& Madrid, 2012; Padial-Ruz, Ibáñez-Granados, Fernández \& UbagoJiménez, 2019). Añadiendo a la Educación Física un contenido que refleja elementos con significados reflexivos más que una simple reproducción del movimiento (Zandoná Júnior \& Silva, 2018).

Las aportaciones que la danza ofrece en la educación, como puede ser el desarrollo de destrezas básicas, de habilidades perceptivo-motoras, de socialización y de la aproximación a sus aspectos históricos, socioculturales y artísticos (Nicolás, Ureña, Gómez \& Carrillo, 2010), aún necesitan lograr la conquista de un espacio real dentro del aula y gran parte de este cometido es de responsabilidad del profesor de Educación Física, ya que la danza como parte del currículum escolar, todavía no parece tener un abordaje efectivo como 
contenido. Por lo que, parece ser un reto la formación de los futuros profesores en esta materia para que se vean capacitados para su correcta implantación dentro del sistema educativo.

En este sentido, el presente trabajo pretende ofrecer una experiencia didáctica que aproxime al futuro profesorado de Educación Física al contenido de las danzas folclóricas desde una perspectiva sociocultural, a modo de favorecer el conocimiento y la formación del mismo para la consecución del currículum.

\section{Estado de la cuestión}

Es cierto que la danza está presente en el currículum de la Educación Secundaria Obligatoria (ESO) en España y que se empieza a apreciar el valor de la misma para la formación integral del ser humano, ya que podría contribuir sobre indicadores de salud mental, como el estado anímico, la autoimagen corporal, la autoestima, las emociones, el respeto al compañero y el bienestar del alumnado (Padilla \& Coterón, 2013), situando la misma como un instrumento muy válido en la vida del individuo, principalmente en etapa escolar.

No obstante, la danza como parte de los contenidos de la asignatura de Educación Física todavía debe ser revisada y sobretodo resaltada para que su implementación sea más eficaz entre el profesorado y no solo un contenido escaso, puntual y desaprovechado como agente educativo (García, Pérez \& Calvo, 2011). Como afirman, Cañabate, Rodríguez y Zagalaz (2016): «la danza todavía no ocupa un espacio con identidad educativa propia» (p.61). Por mucho que se reconozca su importancia y efectividad formativa en la educación, distintos estudios demuestran la poca o ninguna aplicación de la misma entre el profesorado, siendo la falta de formación del docente uno de los principales motivos que no se trabaje este contenido (Cañabate, et al., 2016; Conesa \& Angosto, 2017), y la falta de espacio curricular (Marquis, 2017).

Tal limitación en la formación inicial del profesorado, es explicada por Moreno, Toro y Gómez (2020) como producto de los propios contenidos desarrollados en las universidades en la formación del profesorado de Educación Física, que se centran notablemente en los criterios productivos basados en el logro y dominados por el deporte, obviando los contenidos de carácter más emocionales y expresivos del propio movimiento, posicionando los mismos a un estatus inferior al resto de los contenidos.

El estudio de Yoncalik, Demirel y Yoncalik (2017) constató que, a pesar de ser positiva la percepción de la danza popular como una actividad social, su muestra de 167 profesores de Educación Física y Deportes consideró el baile y el deporte como dos campos diferentes y que la enseñanza de la danza no era un deber que debían cumplir en las escuelas. De la misma manera, se observó que el grupo de profesores especialistas que tuvieron una actitud más positiva hacia la danza popular fueron los maestros de artes marciales. Quizás porque este tipo de deporte está vinculado a las costumbres e historia de determinadas sociedades, coincidiendo con el sentido cultural que viene impregnado en la danza tradicional.
Un medio para que el alumnado entienda el sentido del movimiento, como forma de expresión personal del hombre vinculado a las costumbres y vivencias de una sociedad, podría ser a través de propuestas de trabajo con la danza folclórica en la educación. Ya que favorecería el conocer y experimentar de la danza desde una perspectiva sociocultural, como una herramienta interesante para facilitar la interrelación personal en clase. Por eso mismo, este es uno de los objetivos propuestos por Nicolás et al. (2010) para la danza educativa, para conocer por medio del movimiento los elementos históricos, sociales, culturales y artísticos que la constituyen. Igualmente, Rovegno y Gregg (2007) apuestan por el diseño de programas de danzas populares integradas con temas interdisciplinarios y multiculturales, para que se resalte esta experiencia cultural en la educación. Por otra parte, formadores en danzas folclóricas asocian el concepto de las mismas a aspectos metafóricos de amor, de felicidad, emoción y energía que son elementos esenciales para el ser humano en cualquier etapa de la vida (Karabulut \& Dogan, 2018).

El desarrollo de las danzas folclóricas en el plan curricular se apoya en la competencia de conciencia y expresiones culturales, establecidas en el artículo 2 del Real Decreto 1105/ 2014, de 26 de diciembre de 2014, para el currículo básico de la Educación Secundaria Obligatoria y del Bachillerato. Este contenido permitiría a los alumnos entender elementos de una sociedad y una época, así como estas formas de expresión del sentir y del pensar han ido evolucionando hasta llegar a nuestra actualidad. Para eso, Vieira (2014) cree que los esfuerzos educativos deberían centrarse en la interacción de asignaturas como la Educación Artística y la Educación Física para impulsar la danza como una práctica pedagógica que promueve la acción y la reflexión del estudiante sobre su realidad, desarrollando su sentido cultural y un lenguaje más que corporal que el hombre construye y reconstruye al largo de su historia. Por consiguiente, la danza se refleja en algunas comunidades de España como contenido cultural, danzas históricas y danzas actuales, presentes en el tercero curso de la ESO, dentro del área de Educación Física, acorde el Decreto 48/2015, de 14 de mayo, para el currículo de la Educación Secundaria Obligatoria de la Comunidad de Madrid.

Adicionalmente, se le atribuye a la Educación Física como un campo para mejorar la competencia intercultural en niños y jóvenes, clave para el manejo de la heterogeneidad de la sociedad globalizada de la actualidad. Por eso mismo, Grimminger-Seidensticker y Möhwald (2017) ven de gran ayuda aportar estudios empíricos que demuestren este supuesto. Siendo la danza un contenido de la Educación Física que puede contribuir sustancialmente a esta experiencia cultural, por consiguiente, es una decisión educativa necesaria diseñar y enseñar contenidos didácticos a ese respeto (Rovegno \& Gregg, 2007), uniendo el mundo científico y el folclore a través del desarrollo de danzas culturales e históricas en las escuelas desde una perspectiva amplia e interdisciplinar (Rizo, 1996). Aunque está claro que para una experiencia efectiva será necesario la implicación y la formación previa del profesorado en la enseñanza de la danza folclórica, por lo que hay que mejorar la oferta de cursos formativos y seminarios para que el profesorado pueda llevarlos adecuadamente al espacio educativo (Peña \& Nicolás, 2019). 


\section{Planteamiento didáctico-experiencia}

Partiendo de la importancia que la formación inicial tiene para la futura actuación del profesor de Educación Física en la culminación del plan curricular, se vio la necesidad de aportar mayor instrucción sobre la danza para ayudar en la capacitación del futuro docente y darle soporte en el proceso de enseñanza-aprendizaje de este contenido en el ámbito educativo. Para ello, se ha llevado al aula universitaria la experiencia de trasladar estudiantes del grado de Ciencias de la Actividad Física y del Deporte, a un programa de enseñanza-aprendizaje de danzas folclóricas de distintas sociedades y épocas, para vivir de una forma integral todas estas manifestaciones culturales y comprender como este tipo de expresión puede tener lugar dentro de la educación.

La experiencia ha sido desarrollada en la Universidad Francisco de Vitoria, contando con un total de 108 estudiantes (95 hombres y 13 mujeres), distribuidos en cinco grupos. Todos los participantes se encontraban cursando la asignatura de Actividades Físicas Artístico-Expresivas del curso 2017/2018, impartida en el segundo año de la carrera, que tiene como una de sus finalidades dar herramientas educativas a los futuros profesionales en Educación Física para que puedan conocer, diseñar, desarrollar y reflexionar en los elementos de la expresión corporal, la comunicación no verbal y la danza, que son contenidos correspondientes al currículo de la Educación Secundaria Obligatoria.

La experiencia se llevó a cabo por los tres profesores responsables de la asignatura y ya consolidados en la enseñanza de danzas folclóricas. Previamente a la conformación del proyecto, dichos profesores realizaron una investigación sobre la literatura existente para la formulación de estrategias adecuadas al propósito planteado, considerando algunas fuentes representativas para el estudio (Conesa, 2017; Rovegno \& Gregg, 2007).

Una vez realizada la revisión, los profesores diseñaron el programa adecuando la estructura del mismo a la realidad del currículum de la asignatura. Finalmente, confeccionaron una propuesta didáctica para que el contenido de danzas folclóricas fuera impartido en tres sesiones presenciales con una duración de dos horas cada una, más las horas de trabajo autónomo no presencial que los participantes deberían dedicar para la consolidación del aprendizaje en el tema. La estructura de intervención fue la misma para cada uno de los cinco grupos de estudiantes.

Los recursos materiales utilizados fueron una sala amplia con espejos y adaptada para prácticas físicas, textos científicos, recursos audiovisuales de danzas del mundo, equipo de música y proyector, picas de madera de 120 centímetros, instrumentos musicales (un Atabaque y un Bongó) y disfraces temáticos.

En cada sesión se contempló la aplicación de metodologías activas e integradoras que permitieran a los alumnos involucrarse en el proceso, en mayor o menor medida dependiendo de la tarea. Los profesores prestaron su ayuda durante todas las etapas actuando como facilitadores en el proceso de enseñanza-aprendizaje de los estudiantes. Asimismo, por medio de la observación, descripción en los diarios de campo y de la grabación en vídeo de algunas partes de la experiencia, los profesores pudieron proporcionar un mejor apoyo al alumnado en la resolución de las dudas.

El proceso didáctico ha pasado por un abordaje teórico de los fundamentos, contexto sociocultural y valor educativo de la misma, a la vivencia práctica a través del mando directo del profesorado, culminado en un role play de los estudiantes para potenciar el proceso colaborativo, creativo y reflexivo en el aprendizaje de la danza folclórica, como se detalla a continuación.

\section{$1^{a}$ sesión: Introducción teórica de las danzas folclóricas y su valor educativo}

Esta primera sesión tenía como objetivo principal introducir la teoría con la explicación y exposición del tema, con la ayuda de textos y recursos audiovisuales. Al largo de la sesión, se realizó un foro de discusión en el cual los alumnos fueron interviniendo con dudas, opiniones y debates sobre el valor de la danza para la sociedad y la educación, como enseñarla dentro de la Educación Física y enfocarla desde una perspectiva más amplia que solo la corporal para dar a conocer los elementos socioculturales de una forma plena.

La enseñanza sobre las danzas folclóricas en esa fase y a través de los recursos audiovisuales sirvieron para contextualizar cómo los distintos bailes y ritos conectan el pasado con el presente, con las costumbres y las tradiciones de grupos culturales particulares, y cómo es posible identificar por medio de las danzas y los elementos que las constituyen (indumentaria, música, literatura, historia, geografía, etc.) la forma en que pensamos y actuamos dentro de nuestra cultura, las diferencias con las demás y la importancia de aprender, entender y respetar esas diferencias interculturales.

En este contexto, los contenidos de la danza folclórica presentados por los profesores sirvieron de puente entre el conocimiento sociocultural de la danza con su desarrollo en el entorno educativo, favoreciendo a la interacción de los participantes en un debate para exponer sus opiniones y cómo entendían el valor de la danza en la educación y su sentido sociocultural. También fueron utilizados varios textos científicos para fundamentar la relevancia del tema en las escuelas y para enseñar cómo se presenta en la actualidad la aplicación del contenido entre el profesorado de Educación Física. Un ejemplo es la intervención educativa en un grupo de alumnos realizada por Díaz (2012), que utilizando las danzas folclóricas como vía de conocimiento y aprendizaje de la propia cultura de los discentes ha obteniendo como resultado una valoración elevada sobre el patrimonio y un aumento en la formación integral. También el estudio de Sborquia y Gallardo (2002) apuesta por el contenido educativo y la riqueza cultural que las danzas folclóricas permiten en el ámbito escolar. Entretanto, estos autores creen que no todas las danzas son apropiadas para la enseñanza, pues muchas están descontextualizadas y faltas de valor ético y moral. Dicha reflexión sirve de apoyo para los futuros docentes a la hora de aplicar filtros y determinar aquellos bailes que realmente aportan un significado sociocultural apropiado para la enseñanza en el aula.

\section{$2^{a}$ sesión: Taller de danzas folclóricas de los cinco con- tinentes \\ El objetivo de esta sesión ha sido acercar la teoría previa- mente presentada y discutida a la práctica de un taller con}


diversas danzas folclóricas. Estas fueron seleccionadas por el profesorado para el taller con la intención de invitar a los alumnos al encuentro con un poco de la cultura y la historia de los cinco continentes (Asia, Europa, América, Oceanía y África). Para la selección de las danzas incluidas en el taller se priorizó aquellas que mejor caracterizaban una determinada sociedad y época, para que se pudiera hacer visible los diferentes elementos culturales y su enseñanza desde una perspectiva amplia en la que se podía asociar los movimientos rítmicos y expresivos a componentes de otras disciplinas y así propiciar la interiorización del contenido. Así mismo, la selección de determinados bailes tradicionales se justificó en el planteamiento de incentivar la colaboración grupal, el trabajo de técnicas coreográficas, la creatividad y la libertad de expresión dentro de un contexto, para que los estudiantes universitarios pudieran experimentar distintos métodos de enseñanza del tema.

Previo a la práctica de las danzas, para facilitar el aprendizaje y dar sentido a los movimientos ejecutados, se procedió a dar una breve explicación, con la ayuda de soporte visual, sobre el origen, evolución y significado de los movimientos, relacionando cada danza con peculiaridades sociales, históricas y geográficas, al igual que con la música, el arte, la decoración de una época, el vestuario y los varios elementos que caracterizan las costumbres de un determinado pueblo. En esta línea, Rovegno y Gregg (2007) apuestan por programas de colaboración entre maestros de Educación Física con otras disciplinas para la construcción de unidades interdisciplinarias que resalten las distintas culturas del mundo por medio de danzas populares significativas.

El enfoque integral dado a la experiencia, se justifica en el desarrollo de relaciones interdisciplinarias que profundizan y contribuyen con el perfeccionamiento del proceso de enseñanza aprendizaje y con la formación de profesionales (Llano, et al., 2016; Vieira, 2014), en este caso, siendo abordado los contenidos de la danza folclórica en toda su esencia sociocultural y en la ejecución práctica.

El taller ha buscado la participación y cooperación de todo el alumnado en la ejecución de las varias danzas, sin embargo, debido al carácter integrador del mismo y la valoración a la creatividad de los futuros profesores, se les ofreció la posibilidad de probar con los instrumentos musicales y con el montaje coreográfico de determinados bailes.

La metodología de enseñanza utilizada ha variado dependiendo de la danza y del grado de dificultad apreciado en cada grupo. Por norma general, la secuencia de movimientos ha sido demostrada de manera global, dando énfasis en la repetición solamente para aquellos pasos de mayor grado de complejidad de ejecución, en los que se instruía de una forma más analítica y con el mando directo del profesorado. La disposición de los profesores confluía entre la organización de las coreografías, instrucción y corrección de los pasos, explicaciones, resolución de dudas, control de los espacios y de los materiales.

Asimismo, fueron trabajadas distintas técnicas, dimensiones espaciales, desplazamientos y ritmos propios de cada danza. Aparte de incorporar momentos propicios con metodologías creativas y de aprendizaje cooperativo que permitieron al alumnado la construcción de secuencias coreográficas para explorar la creatividad y la interacción grupal. Este fue el caso de la danza de Oceanía, que proporcionó la libertad de expresión y la improvisación, favoreciendo la autonomía y participación de los estudiantes en la construcción del conocimiento sobre el tema.

Las danzas folclóricas de los cinco continentes seleccionadas por los profesores, se distribuyeron de la siguiente manera:

Asia: calentamiento con una danza divertida, con movimientos característicos de las artes marciales;

Europa: dos danzas en pareja con formación circular, variantes del «Fandango»;

América: danza indígena «Caiapó», con distintos diseños espaciales, desplazamientos y con la utilización de picas de madera para abstraer sonidos;

Oceanía: creatividad en la elaboración de secuencias coreográficas e intensidad en la percusión corporal y expresión facial de la danza «Haka»;

África: Improvisación y libertad de expresión con danzas tribales africanas conectadas a las características de su cultura. Vuelta a la calma y reflexión.

A través de la interiorización de la práctica por el alumnado, los profesores de la asignatura fueron haciendo un feedback con los mismos, para ir percibiendo la receptividad, aceptación y asimilación del contenido del taller y con la ayuda de los vídeos grabados en esa sesión. Los estudiantes estuvieron bastante implicados en todo el proceso del taller, tanto en la ejecución de las técnicas de cada danza como en el trabajo colaborativo para el desarrollo de las secuencias coreográficas creativas de determinados bailes.

\section{$3^{a}$ sesión: Role play en danzas del mundo}

Para no limitar la experiencia a la simple instrucción, reproducción o adquisición de conocimientos, en la tercera sesión los estudiantes tuvieron como objetivo: investigar, seleccionar, desarrollar, reproducir y explicar una danza folclórica de cualquier parte del mundo. A través de la técnica metodológica de role play, se les dio a los participantes la oportunidad de ser ellos los protagonistas en la búsqueda de las danzas folclóricas, sus elementos socioculturales, montaje coreográfico y puesta escénica. Además, eran los responsables de explicar cómo enfocar la danza elegida dentro del ámbito educativo destacando su valor para ser llevado al aula.

En esta fase final de la propuesta y para cada uno de los cinco grupos, los participantes se subdividieron en grupos de cuatro o cinco componentes. Los mismos tuvieron que utilizar las horas no presenciales que conforman la asignatura para realizar una investigación sobre las danzas de distintos países y épocas, elegir un determinado baile, crear secuencias coreográficas, averiguar los contenidos socioculturales de la danza seleccionada, ensayar la misma, buscar la vestimenta y la música acorde. Posteriormente, llevaron a cabo la escenificación de su danza tradicional, así como la explicación a toda la clase sobre la misma.

Todas las danzas presentadas fueron grabadas para que posteriormente los participantes pudieran ver su actuación y reflexionar juntos sobre sus puntos positivos y los que deberían mejorar. No obstante, las grabaciones también sirvieron de instrumento para orientar el profesorado a la hora 
de calificar el desempeño de cada grupo.

Esta actividad de role play realizada por los estudiantes ha contado para la calificación de la asignatura en un total de $5 \%$ de la nota final. Siendo utilizada una rúbrica como instrumento de valoración cuantificable de las danzas folclóricas presentadas. La rúbrica se desglosaba en un 70\% correspondiente a la nota del profesor y un 30\% referente a la propia percepción que cada grupo tenía sobre su correspondiente danza y fundamentación.

Aunque la esencia de la experiencia era la propia vivencia y acercamiento de los estudiantes al mundo de las danzas folclóricas y su formación en el tema, los participantes tuvieron acceso a los vídeos grabados de la segunda y tercera sesión, aparte del material teórico didáctico utilizado en todo el proceso, para que en un futuro puedan servirse de estos recursos como apoyo en el desarrollo del contenido en el contexto educativo.

\section{Reflexiones sobre el aprendizaje}

La danza como herramienta educativa en el trabajo del cuerpo, del movimiento, de la expresión de los sentimientos y de la creatividad, que favorece el autoconocimiento, la relación con uno mismo y con los demás, el aprendizaje y respecto a otras culturas y a sus elementos socioculturales debe ser reconocida y desarrollada de forma consistente y regular en las escuelas. Para ello, es conveniente una formación previa del profesorado desde el ámbito universitario en lo cual, por medio de experiencias didácticas vivenciales de la danza y sus fundamentos, se inicien en la capacitación, diseño, desarrollo y reflexión sobre el tema.

Por medio de la observación de todo el proceso y la reflexión junto a los estudiantes, podemos valorar de forma positiva la consecución del objetivo de aproximar el alumnado en el conocimiento y formación en el contenido de danza folclórica. Además, la estructura diseñada para la experiencia, con sus diferentes actividades y métodos de enseñanza, proporcionaron un ambiente de aprendizaje satisfactorio e instructivo, motivando a los estudiantes a tomar más responsabilidad por su aprendizaje, a promover un trabajo de reflexión y pensamiento crítico sobre los distintos elementos socioculturales que influyen en el movimiento y su aplicación en el ámbito educativo. Las metodologías de enseñanza vivencial de ese tipo permiten la socialización, la expresión y el desarrollo de la capacidad creativa del alumnado, llevándolos a una mayor implicación en su proceso de aprendizaje (García, et al., 2011). Además, proporciona el aprendizaje más efectivo y permanente por medio de experiencias estimulantes, que incitan desafíos en las lecciones y el uso de varios materiales (Garnett, 2005). Eso se veía reflejado en la experiencia, principalmente, en aquellos momentos en que el alumnado era el protagonista del proceso.

A partir de esta experimentación dentro del aula se hizo un acercamiento vivencial de estudiantes universitarios con danzas de distintos continentes, en las cuales se veían reflejados tanto elementos de la propia danza como de la cultura y tradición de un pueblo. La formación del alumnado en el contenido ha permitido que aprendieran no sólo la teoría y la práctica de nuevas danzas, sino de su valor educativo como un instrumento lleno de posibilidades a ser explorado para la formación plena de sus futuros alumnos y el cumplimento curricular. Cumplimiento que todavía carece de la implicación del profesorado, según Conesa y Angosto (2017), debido al desconocimiento o la falta de preparación específica de los docentes, por requerir una preparación sustancialmente mayor del profesorado, y por la falta de tiempo en el calendario académico para impartir todos los bloques de contenidos, por todo ello, el contenido de la danza parece tener una presencia menor en la docencia.

La oportunidad ofrecida a los estudiantes con la presente propuesta, permitió que ellos participaran en la búsqueda, elección y construcción coreográfica de nuevas danzas tradicionales, brindándoles el descubrimiento de movimientos repletos de la singularidad de una cultura. Igualmente, en distintos momentos de la experiencia se beneficiaron de ser los agentes responsables de su propia formación (Garrote D., Garrote C. \& Jiménez, 2016), al tener la libertad de creación y expresión dentro del contexto folclórico. Especialmente, tuvieron la ocasión de empezar a prepararse como futuros profesionales del área en la aplicación del contenido asimilado y vivido, que es realmente importante, ya que la literatura retrata que el principal facilitador de la enseñanza de la danza en Educación Física escolar es la experiencia y formación del docente (Marquis, 2017).

La experiencia teórico-práctico de danzas folclóricas de distintos continentes puede ser una alternativa a considerar para la aproximación de los estudiantes con los elementos socioculturales reflejados en la danza. Igualmente, una posible herramienta educativa para que futuros profesionales del área se sientan preparados para enseñar este contenido en el aula. De ese modo, se favorecerá la construcción de propuestas educativas sustentadas en procesos culturales e identitarios diversos en un marco de respeto y equidad (Gómez \& Hernández, 2010), en el cual, se le ofrecerá al alumnado una formación integral a través de la vivencia de la danza folclórica como una vía de conocimiento y valoración de su propia cultura (Díaz, 2012) y la de otros, además de un instrumento de expresión y comunicación no verbal con un importante valor educativo.

Se espera que la presente propuesta didáctica pueda contribuir para la futura actuación de los participantes con la danza en las escuelas, una vez que se les ofreció la oportunidad de aprender, vivir y asociar la danza folclórica desde una perspectiva sociocultural más amplia al contexto educativo, siendo este un elemento diferenciador de la experiencia. Asimismo, cabe destacar la acogida positiva de los estudiantes universitarios a la propuesta, sobretodo en las etapas prácticas que se veían reforzadas la interacción del grupo, la creatividad, la motivación y el disfrute de la actividad. Hecho que se ve corroborado con la literatura en cuanto a la influencia positiva de las danzas folclóricas en los factores actitudinales y bienestar psicológico y social de estudiantes que la practican (Stivaktaki, Mountakis \& Bournelli, 2010; Zapata, 2017).

Teniendo en cuenta las dificultades a las que todavía se enfrenta el contenido de la danza para ser tematizado en la escuela, junto a la falta de formación específica del profesorado en el tema, es importante reforzar este tipo de aportación empírica y estimular la expansión de experiencias como ésta, para que se reflexione y valore el espacio del contenido en la formación inicial y la futura actuación del docente, y 
quizás, se consiga una mayor representatividad de la danza en el contexto educativo.

\section{Decisiones de acción para la próxima puesta en práctica}

Para futuros estudios e intervenciones se pretende profundizar en la aplicación de la propuesta junto a instrumentos de evaluación cuantitativos para dar a conocer la percepción del alumnado en cuanto a su aprendizaje sobre el tema. También se considera para las siguientes intervenciones revisar los tiempos dedicados a cada sesión didáctica y su posible ampliación.

\section{Conclusiones}

La experiencia con las danzas folclóricas desde una perspectiva sociocultural muestra ser una posibilidad a tenerse en cuenta como recurso didáctico en el contexto educativo. Por lo que se concluye que este tipo de experiencia didáctica es importante para impulsar el conocimiento y la formación de los futuros profesores de Educación Física, proporcionándoles más herramientas para el desarrollo integral del alumnado.

Por último, recalcar la importancia de más estudios relacionados con la enseñanza de la danza en las escuelas y las dificultades enfrentadas por la misma para constituirse como un contenido consolidado reforzarían la relevancia del tema y su consecución en el plan de estudios.

\section{Agradecimientos}

Este trabajo se enmarca en el proyecto de innovación docente «Más allá de la danza» de la Convocatoria de Ayudas a la Innovación Docente 2019 financiado por la Universidad Francisco de Vitoria.

\section{Referencias}

Cañabate, D., Rodríguez, D., \& Zagalaz, M. L. (2016). Educación física y danza: valoración de su integración en las escuelas de primaria de las comarcas de Girona. Apunts. Educación Física y Deportes, 125, 5362.

Conesa, E. (2017). Las danzas del mundo como herramienta formativa. Trances, 9(5),747-780.

Conesa, E., \& Angosto, S. (2017). La expresión corporal y danza en la educación física de secundaria y bachillerato. Cuadernos de Psicología del Deporte, 17(2), 111-120.

Decreto 48/2015, de 14 de mayo, del Consejo de Gobierno, por el que se establece para la Comunidad de Madrid el currículo de la Educación Secundaria Obligatoria. Boletín Oficial de la Comunidad de Madrid. Madrid, 20 de mayo de 2015, núm. 118, pp. 220.

Díaz, A. M. (2012). Intervención en el aula a través de la danza folclórica. Ensayos. Revista de la Facultad de Educación de Albacete, 27, 101108.

Domínguez, C. L., \& Castillo, E. (2017). Relación entre la danza librecreativa y autoestima en la etapa de educación primaria. Cuadernos de Psicología del Deporte, 17(1), 73-80.

Fuentes, A. L. (2006). El valor pedagógico de la danza. Valencia: Universidad de Valencia, Servicio de Publicaciones.

García I., Pérez, R., \& Calvo, A. (2011). Iniciación a la danza como agente educativo de la expresión corporal en la educación física actual. Aspectos metodológicos. Retos. Nuevas Tendencias en Educación Física, Deporte y Recreación, 20, 33-36.

Garnett, S. (2005). Using brainpower in the classroom: five steps to accelerate learning. Abingdon: Routledge.

Garrote, D., Garrote, C., \& Jiménez, S. (2016). Factores influyentes en motivación y estrategias de aprendizaje en los alumnos de grado.
REICE. Revista Iberoamericana sobre Calidad, Eficacia y Cambio en Educación, 14(2), 31-44.

Gil-Madrona, P., Gutiérrez, E. C., \& Madrid, P. D. (2012). Incremento de las habilidades sociales a través de la expresión corporal: la experiencia en clases de iniciación al baile. Cuadernos de Psicología del Deporte, 12(2), 83-88.

Gómez, J. T., \& Hernández, J. G. (2010). Relaciones interculturales, interculturalidad y multiculturalismo: teorías, conceptos, actores y referencias. Revista Cuicuilco, 17(48), 11-34.

Grimminger-Seidensticker, E., \& Möhwald, A. (2017). Intercultural education in physical education: results of a quasi-experimental intervention study with secondary school students. Physical Education and Sports Pedagogy, 22(5), 445-458.

Karabulut, E., \& Dogan, P. (2018). Metaphorical perceptions of folk dances trainers regarding the concept of folk dances. International Journal of Higher Education, 7(2), 84-94.

Llano, L., Gutiérrez, M., Stable, A., Núñez, M. C., Masó, R. M., \& Rojas, B. (2016). La interdisciplinariedad: una necesidad contemporánea para favorecer el proceso de enseñanza aprendizaje. Medisur, 14(3), 320-327.

Marquis, J. (2017). A descriptive profile of dance curriculum in Physical Education teacher education programs (Dissertation-PhD). Department Kinesiology and Health, Georgia State University. EEUU.

Moreno, A., Toro, S., \& Gómez, F. (2020). Formación inicial de maestros de educación física: conectando un quehacer pedagógico decolonial con la intervención social, política e insurgente del espacio público. Retos, Nuevas tendencias en Educación Física, Deporte y Recreación, 37, 605-612.

Nicolás, G. V, Ureña, N., Gómez, M., \& Carrillo, J. (2010). La danza en el ámbito de educativo. Retos. Nuevas Tendencias en Educación Física, Deporte y Recreación, 17, 42-45.

Padial-Ruz, R., Ibáñez-Granados, D., Fernández, M., \& Ubago-Jiménez, J. L. (2019). Proyecto de baile flamenco: desarrollo motriz y emocional en educación infantil. Retos. Nuevas tendencias en Educación Física, Deporte y Recreación, 35, 396-401.

Padilla, C., \& Coterón, J. (2013). ¿Podemos mejorar nuestra salud mental a través de la danza?: una revisión sistemática. Retos. Nuevas Tendencias en Educación Física, Deporte y Recreación, 24, 167-170.

Peña, V. J., \& Nicolás, G V. (2019). Danza en educación infantil: opinión de los docentes. Retos. Nuevas tendencias en Educación Física, Deporte y Recreación, 36, 239-244.

Porpino, K. (2018). Dança é educação: interfaces entre corporeidade e estética ( $2^{\mathrm{a}} \mathrm{ed}$.). Natal, RN: EDUFRN.

Real Decreto 1105/2014, de 26 de diciembre, por el que se establece el currículo básico de la Educación Secundaria Obligatoria y del Bachillerato. Boletín Oficial del Estado. Madrid, 3 de enero de 2015, núm. 3, pp. 172.

Rizo, G (1996). La enseñanza de los bailes y las danzas tradicionales en la escuela: un enfoque interdisciplinar. Eufonía: Didáctica de la Música, 3, 73-84.

Rovegno, I., \& Gregg, M. (2007). Use of folk dance and geography to teach interdisciplinary and multicultural topics: a school-based study. Physical Education and Sports Pedagogy, 12(3), 205-223.

Rueda, B., \& López, C. E. (2013). Música y programa de danza creativa como herramienta expresión de emociones. Retos. Nuevas Tendencias en Educación Física, Deporte y Recreación, 24, 141-148.

Sborquia, S. P., \& Gallardo, J. S. (2002). As danças na mídia e as danças na escola. Revista Brasileira de Ciências do Esporte, 23(2), 105-118.

Stivaktaki, C., Mountakis, C., \& Bournelli, P. (2010). The effect of a cross curricular study programme in Physical Education on the attitudes and perceptions of greek children towards traditional (folk) dance in the first year of secondary school. Research in Dance Education, 11(3), 193-211.

Vieira, M. S. (2014). A danca na arte e na educação física: diálogos possíveis. Revista Tempos e Espaços em Educação, 13, 177-185.

Yoncalik, O., Demirel, M., \& Yoncalik, M. (2017). Perceptions of physical education and sports teachers towards folk dance in Turkey. South African Journal for Research in Sport, Physical Education and Recreation, 39(3), 189-205.

Zandoná Júnior, A., \& Silva, F. H. (2018). Reflexões acerca do currículo e metodologia da Educação Física no Instituto Federal de Goiás: a experiência com materiais didáticos. Retos. Nuevas Tendencias en Educación Física, Deporte y Recreación, 34, 337-342.

Zapata, A. (2017). Bienestar psicológico y bienestar social en estudiantes universitarios que realizan danzas folklóricas (Tesis Doctoral). Pontificia Universidad Católica del Perú. 